\title{
BMJ Open Peer-led HIV care and the UNAIDS 90- 90-90 treatment targets in Tigrai, Ethiopia: a cluster randomised trial and economic evaluation of teach-test-link- trace model (TTLT) trial protocol
}

\author{
Hailay Gesesew (D) , ${ }^{1,2}$ Paul Ward, ${ }^{2}$ Jonathan Karnon, ${ }^{2}$ Richard Woodman, ${ }^{2}$ \\ Lillian Mwanri
}

To cite: Gesesew H, Ward P, Karnon J, et al. Peer-led HIV care and the UNAIDS 90-9090 treatment targets in Tigrai, Ethiopia: a cluster randomised trial and economic evaluation of teach-test-link-trace model (TTLT) trial protocol. BMJ Open 2021;11:e043943. doi:10.1136/ bmjopen-2020-043943

- Prepublication history for this paper is available online. To view these files, please visit the journal online (http://dx.doi. org/10.1136/bmjopen-2020043943).

PW and LM contributed equally.

Received 20 August 2020 Accepted 12 May 2021

Check for updates

(c) Author(s) (or their employer(s)) 2021. Re-use permitted under CC BY-NC. No commercial re-use. See rights and permissions. Published by BMJ.

${ }^{1}$ Epidemiology, Mekelle University, Mekelle, Ethiopia ${ }^{2}$ Public Health, College of Medicine and Public Health, Flinders University, Adelaide, South Australia, Australia

Correspondence to

Dr Hailay Gesesew;

hailushepi@gmail.com

\section{ABSTRACT}

Introduction This study will evaluate the acceptability and effectiveness of a new intervention programme called teach-test-link-trace (TTLT) model in Tigrai, Ethiopia, where peer educators counsel about HIV (teach), perform house-tohouse HIV testing (test) through pinprick HIV testing $(\mathrm{H} 2 \mathrm{H})$ or OraQuick HIV self-testing (HIVST), link HIV-positive patients to HIV care (link) and trace lost patients house-to-house (trace). Methods and analysis The four-arm cluster randomised trial will be conducted in five phases: Phase 1 will assess the acceptability of the TTLT model using a cross-sectional survey among adults aged 18 years irrespective of HIV status in 6909 households from 40 villages (kebeles). Phase 2 will assess effectiveness of the TTLT model through comparing intervention and control groups among HIV negative or unknown HIV status. The intervention groups will receive one of the three home-based interventions provided by peer educators: (1) demonstrate and distribute OraQuick HIV self-testing kits (HIVST), (2) perform pinprick HIV testing $(\mathrm{H} 2 \mathrm{H})$ and $(3)$ offer a choice to either receive HIVST or $\mathrm{H} 2 \mathrm{H}$. The control group will receive the standard care in which nurses counsel and refer eligible household members to nearby health facilities to access existing HIV testing services. The primary outcomes of the interventions are proportion of individuals who know of their HIV status (first 90), link to HIV care and treatment (second 90) and meet virological suppression (third 90). We will perform process evaluation through qualitative interviews in phase 3 , economic evaluation for cost-effectiveness analysis in phase 4 and a sustainability exit strategy using nominal group technique in phase 5 . We will apply descriptive and inferential statistics for quantitative studies, and thematic framework analysis for qualitative studies.

Ethics and dissemination Ethics approval was obtained from the Southern Adelaide Clinical Health Research Ethics Committee (SAC HREC), South Australia, and findings will be disseminated through publications, conference presentations, media release and policy briefs.

Trial registration number ACTRN12620000570987p.

\section{BACKGROUND}

Ethiopia is one of the sub-Sahara African countries that is disproportionally affected. ${ }^{1}$

\section{Strengths and limitations of this study}

- This is the first cluster randomised trial designed to assess the acceptability, clinical and costeffectiveness of peer educators-led HIV care services in Ethiopia.

- The findings will allow assessment of the acceptability, feasibility and effectiveness of home-based HIV self-test and immediate HIV testing to increase testing uptake, treatment linkage and treatment outcomes.

- The results will provide evidence on whether the peer-led HIV care services is acceptable and is clinically and cost-effective.

- There may be safety concerns of stress, anxiety or self-harm associated with knowing the positive HIV status.

- The clusters may be small given the number of expected outcomes and the randomisation process may insufficiently reduce the sampling bias.

The most recent data inform that the HIV prevalence in Ethiopia was between $0.1 \%$ and $4.8 \%$ in $2017 / 2018 .^{2}$ In 2016, there were an estimated 36990 new HIV infections, 670906 people living with HIV and 20000 AIDSrelated deaths. ${ }^{3}$

To halt this epidemic, Ethiopia, like other countries, subscribed to the UNAIDS 90-90-90 treatment targets, a programme that was launched in Melbourne, Australia, in 2014. ${ }^{4}$ The targets aimed at diagnosing $90 \%$ of people living with HIV (UNAIDS first 90 ), providing $90 \%$ of those diagnosed with antiretroviral therapy (ART) (UNAIDS first 90) and achieving viral suppression for $90 \%$ of patients receiving treatment (UNAIDS first 90) by 2020. Given most countries, including Ethiopia, ${ }^{5}$ fail to meet the target by 2020, the fast track targets are extended to UNAIDS 
95-95-95 to be attained in $2030 .{ }^{6}$ To meet the targets for Ethiopia, this study aims to test a new and comprehensive intervention programme, never been implemented in Ethiopia before, called the teach-test-link-trace (TTLT) model. The TTLT model involves HIV-positive volunteer patients (peer educators) who do HIV counselling (teach), perform home-based HIV testing (test) through HIV self-testing (HIVST) and pinprick house-to-house HIV testing (H2H), link HIV-positive patients to HIV care (link) and trace lost patients house-to-house (trace). In the Ethiopian setting, peer educators are trained HIV-positive individuals who publicly disclose their HIV status. ${ }^{7}$

Although not extensively explored, global evidence shows that peer educators make a substantial contribution to HIV testing uptake. For example, studies in Africa such as Kenya, Tanzania and Mozambique have shown that the peer educators and/or community health workers have brought significant improvements to the achievements of the UNAIDS 90-90-90 targets. ${ }^{8}$ Additionally, a study in Zimbabwe assessed the impact of peer educators on improving HIV testing uptake through HIVST and was found effective. ${ }^{10}$ Evidence from Uganda and Zambia showed that individuals accept and prefer HIVST than the routine facility-based HIV testing. ${ }^{11}$ A systematic review in low-income and middle-income countries found that home-based HIV testing was found to increase HIV testing uptake. ${ }^{12}$

It has to be acknowledged that the impact of peer educators is beyond HIV testing, as they have also shown to improve the treatment linkage. For example, in Vietnam, a pilot study found that peer educators significantly helped people to know their HIV status and start on treatment for those diagnosed with HIV. ${ }^{13}$ This pilot study in Can Tho, Vietnam, reported that 2520 people were tested, 140 (5.6\%) were found to be HIV positive and 131 $(93.6 \%)$ of those who were diagnosed with HIV started the treatment. Evidence also revealed that the peer-led HIV care services were reported to be cost-effective and comprehensive intervention. ${ }^{14}$

There has not been, however, a study that assessed the effectiveness of peer-led HIV testing through the novel testing strategies, HIVST and $\mathrm{H} 2 \mathrm{H}$, and their impact on improving the treatment linkage and virological suppression in Ethiopia. The study aims to test whether peer educators-led delivery of $\mathrm{H} 2 \mathrm{H}$ and HIVST approaches in Tigrai, Northern Ethiopia, can increase the proportion of people who know their HIV status, link to treatment and achieve virological suppression. The trial also aims to assess a number of important safety endpoints including HIV-related stigma, and the cost-effectiveness and sustainability exit strategy of the new programme.

\section{METHODS AND PARTICIPANTS \\ Study protocol}

The protocol for this trial is registered (ACTRN12620000570987p) in the WHO International Clinical Trials Registry Platform (ICTRP) of Australia and New Zealand Clinical Trials Registry (ANZCTR).

\section{Study setting}

The project will be conducted in Tigrai regional state located $780 \mathrm{~km}$ North of the capital Addis Ababa, Ethiopia, with a total population of 8.3 million people. The administrative system in Ethiopia has a hierarchical structure including the top most, the federal level, to the most bottom, the kebele (village) level. The organisational structure and health systems tier of Ethiopia is described in the Ethiopian Health Sector Development Programme (figure 1). ${ }^{15}$ From the regional level where this study will take place, the administrative system structure comprises regional state, zones, woreda (districts) and kebele.

Tigrai is a regional state with 7 zones, 80 districts and 799 kebeles. A kebele is the lowest administrative unit with an average of 5000 people (1000 households). Served by two community health workers, the health post is the lowest health institutional structure in the rural areas and serves a kebele with an average of 5000 people. Five kebeles (five health posts) form a health centre and the health centre caters for total population of 15000 to 25000 people (average of 5000 households). The health centre plays a significant role in coordinating the five

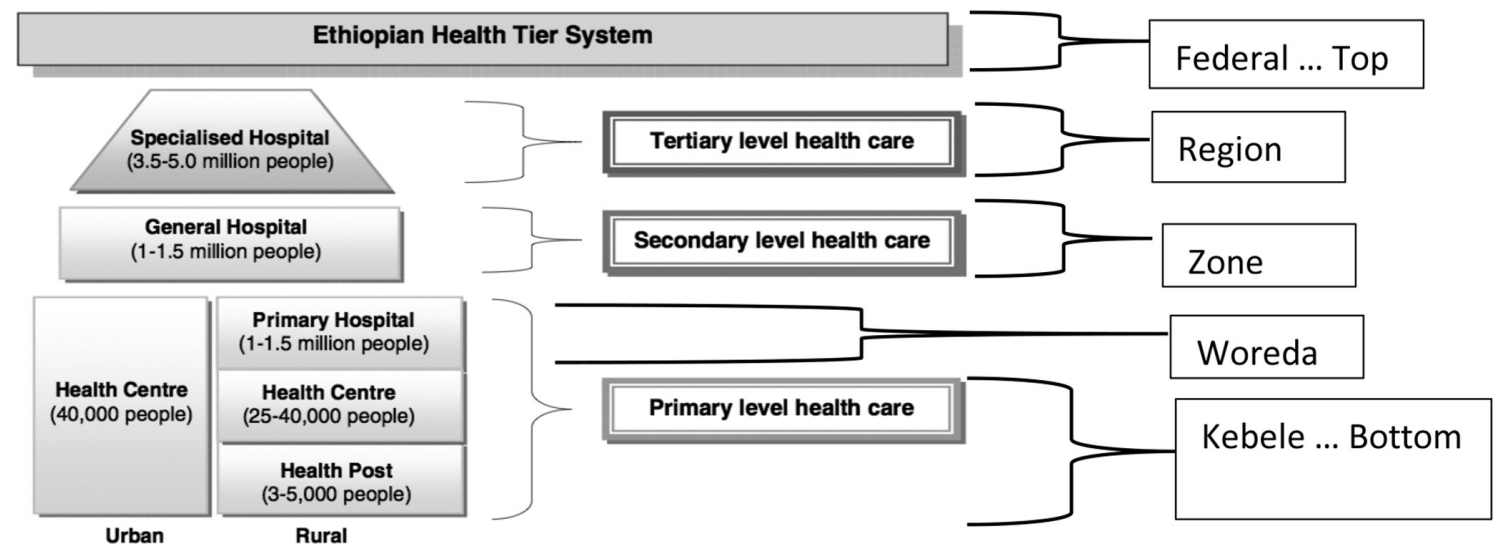

Figure 1 Ethiopia organisational structure and health tier system. This figure presents the organisational structure of Ethiopia from top (federal) to bottom (kebele or village) levels and the three-tier health system. 


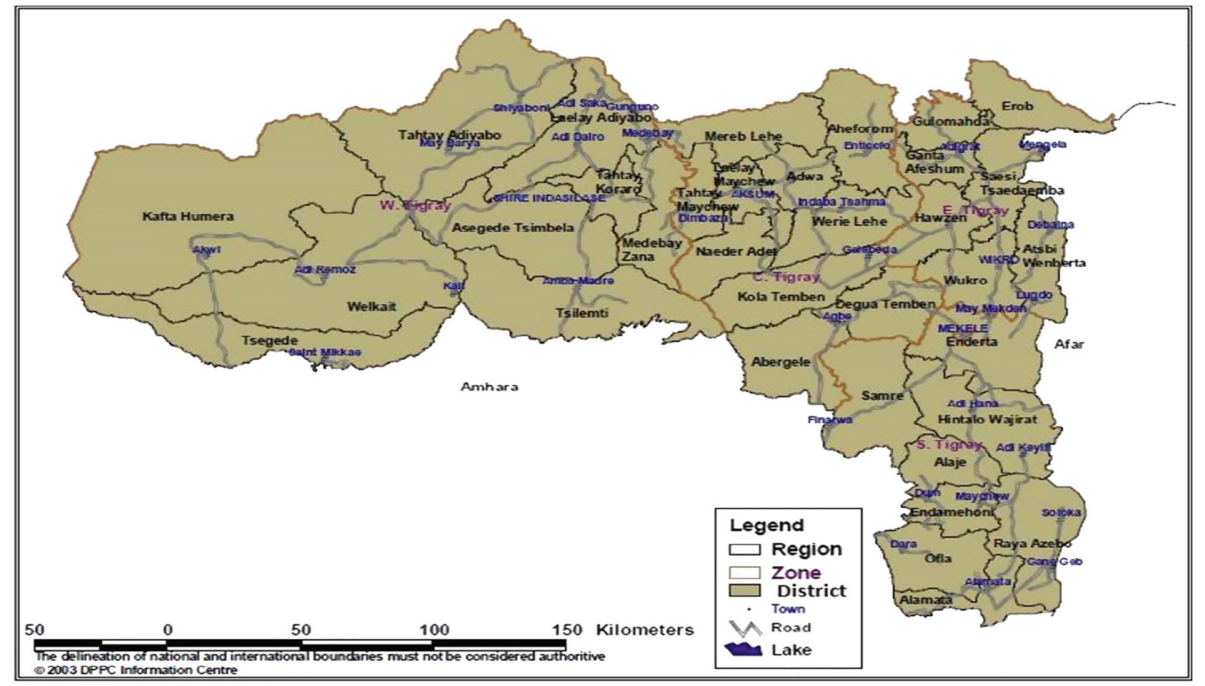

Figure 2 Map of Tigrai, Ethiopia. This figure presents the map of Tigrai, Northern Ethiopia.

health posts in the rural areas. Unlike the rural setting, urban health centre serves up to 40000 people. Tigrai has 39 public hospitals (2 specialised hospitals, 15 general hospitals and 22 primary hospitals), 216 health centres, 500 private health facilities (hospital, clinic and medical centres) and 712 health posts. ${ }^{16}$ Every household in the kebele has a family folder in their health post catchment area, describing the basic demographic characteristics of the household members.

The prevalence of HIV in Tigrai in 2017/2018 was 1.2\%, a proportion higher than the national average $(0.9 \%){ }^{2}$ The prevalence of HIV in Tigrai is higher among women (vs men), urban (vs rural) and commercial sex workers (vs the general population). For example, the prevalence of HIV among women and men was $1.5 \%$ and $0.7 \%$, respectively. ${ }^{2}$ There are 147 health facilities providing HIV testing and treatment services in Tigrai. Recently, 13 health facilities started to provide an oral HIV self-testing service. Each health facility providing HIV care and treatment services in Tigrai has one to five peer educators, who are trained and conduct counselling for noncompliant people diagnosed with HIV. The trial will be conducted in 40 kebeles and 8 health centres, randomly selected from all 7 zones in Tigrai (figure 2). The Tigrai capital, Mekelle, with a total population of 310436 , will be the study headquarters to coordinate all sites.

\section{Aims}

The principal aim of this study is to (1) assess the acceptability and feasibility of the TTLT model; (2) test TTLT model's clinical and cost-effectiveness of HIV diagnosis, ART linkage and virological suppression; and (3) design a sustainable exit strategy of the programme. In addition, the trial also aims to assess: (4) quality of life of HIV patients and (5) HIV-related stigma.

\section{Study design and population}

The TTLT model trial uses a four-arm parallel clusterrandomised controlled design (CRT) including three intervention groups and a control group as described in the study procedures below. The trial (figure 3) will be conducted in five phases.

Phase 1 will be an assessment of acceptability of the model. The population in this phase includes individuals age 18 years and above irrespective of their HIV status selected from participating households.

Phase 2 will be the intervention phase comprising three intervention arms, namely, peer-led house-to-house HIV self-testing (HIVST), peer-led house-to-house pin-prick HIV testing $(\mathrm{H} 2 \mathrm{H})$ and a control arm, standard of care. The target population in this phase are people whose HIV status is unknown or negative.

Phase 3 will be process evaluation of the intervention using qualitative interviews, and the population for this will be a selected individual who participated in the intervention phase. Phase 4 will be cost-effectiveness assessment of the intervention model using economic evaluation. All participants included in the intervention phase will be included in this phase.

Phase 5 will be assessment of programme sustainability after exiting the trial using nominal group technique (NGT). HIV experts in Ethiopia will be the population for the NGT. The trial will start in January 2021 and be completed in December 2022.

\section{Outcomes and measurement}

The primary outcomes of the trial are as follows:

1. Acceptability of the TTLT model-This will be measured through face-to-face interview using Sekhon and colleagues' theoretical framework for acceptability of healthcare interventions. ${ }^{17}$

2. Change in knowledge of HIV status (UNAIDS first 90) - This will be measured through face-to-face interview at enrolment of the trial, followed by HIV testing (diagnosis period) through HIVST and H2H. In OraQuick HIV self-testing, ${ }^{18}$ the test is positive if a T-Line appears and negative if a C-Line appears in the test 
Tigrai region stratified in 7 zones and 746 villages or Kebeles(clusters)

Sustainability exit strategy

Understanding the scalability, defining targeted policy scenarios and suggesting modality to fill the gap

Figure 3 CONSORT (Consolidated Standards of Reporting Trials) flow diagram of trial design. This figure shows the flowchart of trial and including the study enrolment, randomisation and intervention arms for TTLT (teach-test-link-trace) model study. ART, antiretroviral therapy; H2H, peer educators-led pinprick house-to-house HIV testing; HIVST, peer educators-led OraQuick HIV self-testing; LTFU, lost-to-follow-up.

stick. In the pinprick HIV testing, the test is positive if the peer educators report 'reactive' and negative if the peer educators report 'non-reactive' based on the national HIV testing guidelines.

3. Compare proportion of individuals who will be linked to HIV care (UNAIDS second 90) between those who are diagnosed through intervention and control groups-This will be measured at 0 month (enrolment at ART) through extracting their linkage of care from clinical registry. 'Linked to HIV' care refers to enrol- ment of HIV-positive person in to HIV care setting to receive ART or other HIV care services. ${ }^{4}$

4. Compare proportion of individuals who will achieve virological suppression (UNAIDS third 90) between those who are diagnosed through intervention and control groups-This will be measured at 3, 6 and 9 months post ART initiation through extracting viral counts from clinical registry (prospective cohort). Virological suppression refers to viral load test results of $\leq 1000$ copies/mL with two consecutive measurements 
in a 3-month interval after 3 months of ART initiation, that is, at 6 and 9 months after ART initiation. ${ }^{19}$

The secondary outcomes of the interventions are as follows:

1. Health-related quality of life (HRQoL) - This will be measured through facility-based cross-sectional survey using the 31-item WHO-QoL-BREF Tigrigna version ${ }^{20}$ at 3 and 6 months after ART initiation.

2. HIV-related stigma-This will be measured through facility-based cross-sectional survey using 12-item HIVrelated stigma scale ${ }^{21}$ translated to Tigrigna at 3 and 6 months after ART initiation and qualitative interviews.

\section{Sample size determination}

In total, 40 kebeles (clusters) will be recruited using simple random sampling, of which 10 clusters each will be randomly assigned to one of the four study groups. We calculated the sample size based on the primary endpoint of the cluster randomised trial (figure 3) using the Hayes and Bennett formula ${ }^{22}$ shown below:

$$
C=1+\frac{\left(Z_{\alpha / 2}+Z_{\beta / 2}\right)^{2}\left[\left(P_{0}\left(1-P_{0}\right)\right)+\left(P_{1}\left(1-P_{1}\right)\right) / n+k^{2}\left(P 0^{2}+P 1^{2}\right)\right]}{\left(P_{0}-P_{1}\right)^{2}}
$$

(Equation 1)

where $\mathrm{c}$ is the number of clusters per arm, $\mathrm{p}_{0}$ and $\mathrm{p}_{1}$ are estimates of HIV testing uptake in control and intervention clusters, $\mathrm{n}$ is the number of households per cluster and $\mathrm{k}$ is the coefficient of variation of outcomes. Considering $\mathrm{c}$ is $10, \mathrm{p}_{0}$ is $72 \%$ (assumed number of people who knew their HIV status in the control group), ${ }^{23} \mathrm{p}_{1}$ assumes an absolute increase of $15 \%$ using HIVST (intervention 1) and $\mathrm{H} 2 \mathrm{H}$ (intervention 2) and 20\% using HIVST or $\mathrm{H} 2 \mathrm{H}$ (intervention 3) compared with $\mathrm{p}_{0}$, $\mathrm{k}$ of 0.25 and a type I error probability of 5\%, it comes to 124 households per cluster for HIVST and $\mathrm{H} 2 \mathrm{H}$, and 66 households for HIVST or H2H. Adding a 10\% dropout rate, the sample size per cluster will be 136 households HIVST and $\mathrm{H} 2 \mathrm{H}$ and 73 households for HIVST or H2H. Accordingly, the total sample in the trial will be 5440 households for HIVST and H2H and 2920 households for HIVST or H2H. Finally, we take the sample of 5440 households, the maximum sample size, to improve the inference. Multistage sampling technique will be applied, and design effect is needed to increase the precision. We calculated DEFF (design effect) using the following formula:

$$
D E F F=1+(m-1) P \quad \text { (Equation } 2)
$$

where $m$ is the size of clusters and $\rho$ is the intraclass correlation. We have 10 cluster per arm and assumed 0.03 of intraclass correlation, and this comes to a DEFF of 1.27 . The total sample size would be $5440 * 1.27=6909$ households. This sample size will be for primary outcomes 'Acceptability' and 'knowledge of HIV status'. For primary outcomes 'Linkage' and 'Virological suppression' and secondary outcomes (QoL and HIV-related stigma), it is estimated based on the current prevalence rate of HIV in Tigrai, that is, $1.2 \% * 6909=83$. The sample size and sampling technique across study arms, clusters,
Table 1 Sample size and sampling technique for clusters, households and individuals across four study arms of the

\begin{tabular}{|c|c|c|}
\hline Entity & $\begin{array}{l}\text { Sample size included for } \\
\text { trial }\end{array}$ & $\begin{array}{l}\text { Sampling } \\
\text { technique }\end{array}$ \\
\hline Region & $\begin{array}{l}1 \text { region with a total of } 7 \\
\text { zones }\end{array}$ & Purposely \\
\hline Strata & $\begin{array}{l}7 \text { zones from a total of } 746 \\
\text { possible clusters (kebeles) }\end{array}$ & $\begin{array}{l}\text { Stratified } \\
\text { sampling } \\
\text { technique }\end{array}$ \\
\hline $\begin{array}{l}\text { Clusters per } \\
\text { zone }\end{array}$ & $\begin{array}{l}40 \text { clusters in total to be } \\
\text { selected proportional to their } \\
\text { size }\end{array}$ & $\begin{array}{l}\text { Simple } \\
\text { random } \\
\text { sampling }\end{array}$ \\
\hline $\begin{array}{l}\text { Clusters per } \\
\text { study arm }\end{array}$ & $\begin{array}{l}\text { Total clusters included for trial } \\
\text { divided by } 4 \text { (number of study } \\
\text { arms), n=10 clusters per study } \\
\text { arm }\end{array}$ & $\begin{array}{l}\text { Random } \\
\text { assignment }\end{array}$ \\
\hline $\begin{array}{l}\text { Households } \\
\text { per cluster }\end{array}$ & $\begin{array}{l}\text { Total number of households } \\
\text { divided by } 4 \text { (number of study } \\
\text { arms) divided by } 10 \text { clusters, } \\
n=136 \text { households per cluster }\end{array}$ & $\begin{array}{l}\text { Systematic } \\
\text { random } \\
\text { sampling }\end{array}$ \\
\hline $\begin{array}{l}\text { Individuals } \\
\text { per household }\end{array}$ & One person per household & $\begin{array}{l}\text { Simple } \\
\text { random } \\
\text { sampling }\end{array}$ \\
\hline
\end{tabular}
TTLT model study, Tigrai, Ethiopia, 2020-2024

and households and their individual representatives are described in table 1. For qualitative interviews, we will include about 10 participants each from the HIVST, H2H and standard care study groups. We will include 10-15 experts for the NGT.

\section{Randomisation and blinding}

Because each zone has its own peculiarity, 40 clusters (kebeles) from 7 zones will be selected randomly with probability proportional to size, with the largest of the kebeles also being selected with certainty. Prior to randomisation, we will organise a randomisation ceremony and invite local governments, community leaders, community health extension workers and members of the local data monitoring committee (DMC), and this process enhances recruitment strategies and aimed at ensuring the blinding of the participants. The kebeles will be randomly assigned to one of the four study groups. Households will be the intervention recipients and all eligible individuals in the households will be included in the analysis.

To enhance allocation concealment, the peer educators and household participants will not be told the hypothesis of the research question so that to they are blinded to knowledge of the other interventions being conducted. However, they are unblinded to the actual treatment they are receiving.

\section{Eligibility criteria and recruitment}

The research assistant will assess household eligibility by initially screening the households in a kebele using a family folder available in the health post. The research assistant checks if a household has at least one adult 
person aged 18 years old and consider eligible for inclusion. Eligible households will then be selected from each kebele using computer-generated random numbers. The research assistant will also recruit peer educators from each health centre located in selected clusters. To be included in the intervention, peer educators will need to: (1) be literate (primary school and above) because they will interview participants using structured questionnaire; and (2) willing to attend a 2-day training. Peer educators will be excluded if they: (1) do not want to disclose their HIV status during the HIV counselling and testing process, (2) are not fit to conduct home-to-home visit and (3) are not willing to participate in the trial.

A list of the houses of those kebeles assigned to either HIVST, H2H or HIVST/H2H will be provided to peer educators, and a list of households of kebeles assigned to standard care will be provided to nurses. The peer educators or nurses will go to the kebeles and select one person from each of the selected households who fulfil the following inclusion criteria: aged 18 years and above at enrolment, do not know their HIV status or self-report HIV-negative within 3 months before enrolment, able to provide written informed consent, and willing to participate in the assessment at $0,3,6$ and 9 months if positive for HIV when tested. This process will be continued until an eligible person is recruited. A person who is not willing, not able to answer to interview questions or seriously sick will be excluded from the study.

\section{Study procedures}

In the acceptability study, phase 1 , we will know whether study participants had history of HIV testing in the previous 3 months and knew their HIV status through interview. Study participants who will report HIV negative or unknown HIV status will be nominated for the next phase, the intervention phase. This will also be a baseline data for the intervention phase. The study procedure for the control and intervention groups is described in detail below.

\section{Control groups: existing health service standard care}

The control group will receive the existing standard care of HIV testing and ART services provided by health facility. HIV testing will be performed using finger-prick diagnostic tests using the national HIV testing algorithm. In this study group, nurses will visit a household once to counsel participants, distribute condom and refer them to standard HIV testing and care clinics to a nearest (or their preferred) health facility. The information about HIV counselling provided by the nurses to participants under the standard care group will be similar to that provided by the peer educators to participants under the three study intervention groups. Each participant will be provided a coded coupon so that their HIV status, clinical, immunological and virological characteristics at 0,3 , 6 and 9 months (if HIV positive and/or linked to HIV care) can be extracted. We have included nurses in the SOC, as opposed to peer educators. This is to assess the cost-effectiveness of peer educators versus trained health professionals (ie, nurses). Furthermore, nurses in Ethiopian healthcare system perform out of health facility HIV testing routinely to maximise HIV testing coverage and improve detection rate especially in camping periods.

\section{Intervention groups: HIVST or H2H \\ Peer educators}

As described, peer educators are already situated in health facilities to support other patients with HIV. To be involved in the current study, we will provide them a 2-day training about the study aim and study procedures. We will train them by providing HIV counselling contents and techniques on how to conduct $\mathrm{H} 2 \mathrm{H}$ and HIVST, interpret the results, linkage to services for confirmatory testing and ART initiation for reactive results according to the national guidelines. We will also train and prepare them about what to anticipate and how to respond should they encounter events or incidents that may lead to social harms and emotional disturbances. Finally, we will train them on how to record data, handle and store kits. The project team will provide package of kits for $\mathrm{H} 2 \mathrm{H}$, OraQuick HIV Self-Test for HIVST, condom, questionnaire and note pad.

Intervention 1: peer-led house-to-house HIV self-testing (HIVST) In this study arm, peer educators will perform the following steps: travel to each eligible household, randomly recruit eligible person, describe the HIVST procedure, perform pretest counselling, demonstrate HIVST procedure and distribute a coded coupon and oral HIVST kit package. The package kit will contain OraQuick, instructions translated into Tigrigna (local language) and contact address of the peer educator. The written instructions will demonstrate a step-by-step guide on how to use and interpret the results, and are presented by pictures. Participants will be asked whether they want to be supervised or not during the self-test process. The peer educators will also be informed whom to contact should the individuals raise concerns or issues related to the intervention, such as adverse reactions. Peer educators will finally be needed to inform each participant to report to nearby (or their preference) health centre to confirm the HIV status if the result is reactive, and return the HIVST package and coded coupon even if the result is non-reactive. Alternatively, they can contact the peer educators through contact address included in the package should they do not want to go to the health facility. A detail information on how these individuals return the used test kits and whom to contact when they go for confirmatory test will be provided to the study participants.

Intervention 2: peer-led house-to-house pin-prick HIV testing ( $\mathrm{H} 2 \mathrm{H})$ Similarly, in this study arm, the peer educator will travel to each eligible household, then randomly recruit eligible person, describes the $\mathrm{H} 2 \mathrm{H}$ procedure, perform pretest counselling, perform rapid HIV testing through fingerprick of whole blood, interpret the result and provide 
post-test counselling. In order to prevent (fear of) contamination, a laboratory technician will be hired for each peer educator to take a blood sample and provide the sample to the peer educator. The peer educator will interpret the rapid diagnostic result and perform posttest counselling irrespective of the HIV status. The laboratory technician will have no other than the role of taking a sample of blood from the eligible person.

\section{Intervention 3: HIVST or $\mathrm{H} 2 \mathrm{H}$}

In this study arm, peer educators will offer individuals a choice of HIVST or H2H services, and either of the above procedures for the chosen intervention will be carried out.

In all three interventions, the HIV status of participants will be known through HIVST or H2H. This is a diagnosis period where the first outcome, the first UNAIDS 90, will be measured. Peer educators will briefly meet and counsel individuals who will be diagnosed with HIV before they start ART. If a participant does not want to disclose their HIV status to a (particular) peer educator, the participant will be given alternative option such as visiting another peer educator or HIV care provider over the study period.

The participants who are diagnosed with HIV will report (the HIVST group) or be referred (H2H) to HIV care services for ART initiation. The peer educators will then make sure that those participants diagnosed with HIV would start ART or are in HIV care registry in the nearby (or other) health facility. This is a linkage or enrolment in ART care period $(0$ month), the point of time the second outcome (second UNAIDS 90) is measured. Moreover, peer educators will follow those participants at 3, 6 and 9 months after ART to measure viral load, the third outcome (third UNAIDS 90). During the follow-up, the peer educators will make sure if the patients are taking their treatment without interrupt, trace if they discontinue.
If the result in any of the intervention phases is nonreactive, the person will be informed to perform other test after 3 months or be safe from risky sexual behaviours depending on the risk condition of the person. Moreover, counselling will be provided on HIV prevention-related issues.

\section{Data collection process and timelines}

The trial data collection process has multiple phases, and the trial timeline is described in detail in figure 4 .

\section{Acceptability survey and pilot test}

In this phase, we will collect data for acceptability of the TTLT model using a structured questionnaire. The questionnaire covers several topics including demographics, recent HIV testing history (within 3 months before data collection), awareness, attitudes and questions related to acceptability interventions in health research. Using Sekhon and colleagues' theoretical framework for acceptability of healthcare interventions, ${ }^{17}$ we will collect data on the following constructs for acceptability of the TTLT model: burden, affective attitude, ethical consequences, opportunity costs, experience and intention. We will employ a pilot study in 100 households (25 households per arm) from four clusters to assess the financial, technical, administrative and logistic feasibility of the fullscale trial. ${ }^{24}$ The pilot study will also help to revise the power calculations required for the full-scale CRT. Clusters included in the pilot study will not be included in the main trial.

\section{Intervention phase}

We have described the measurement of primary and secondary outcomes above. We will collect data for secondary outcomes such as QoL and stigma. QoL will be measured using a Tigrigna version (local language) of WHOQOL-HIV BREF tool. ${ }^{20}$ The tool has 31 items

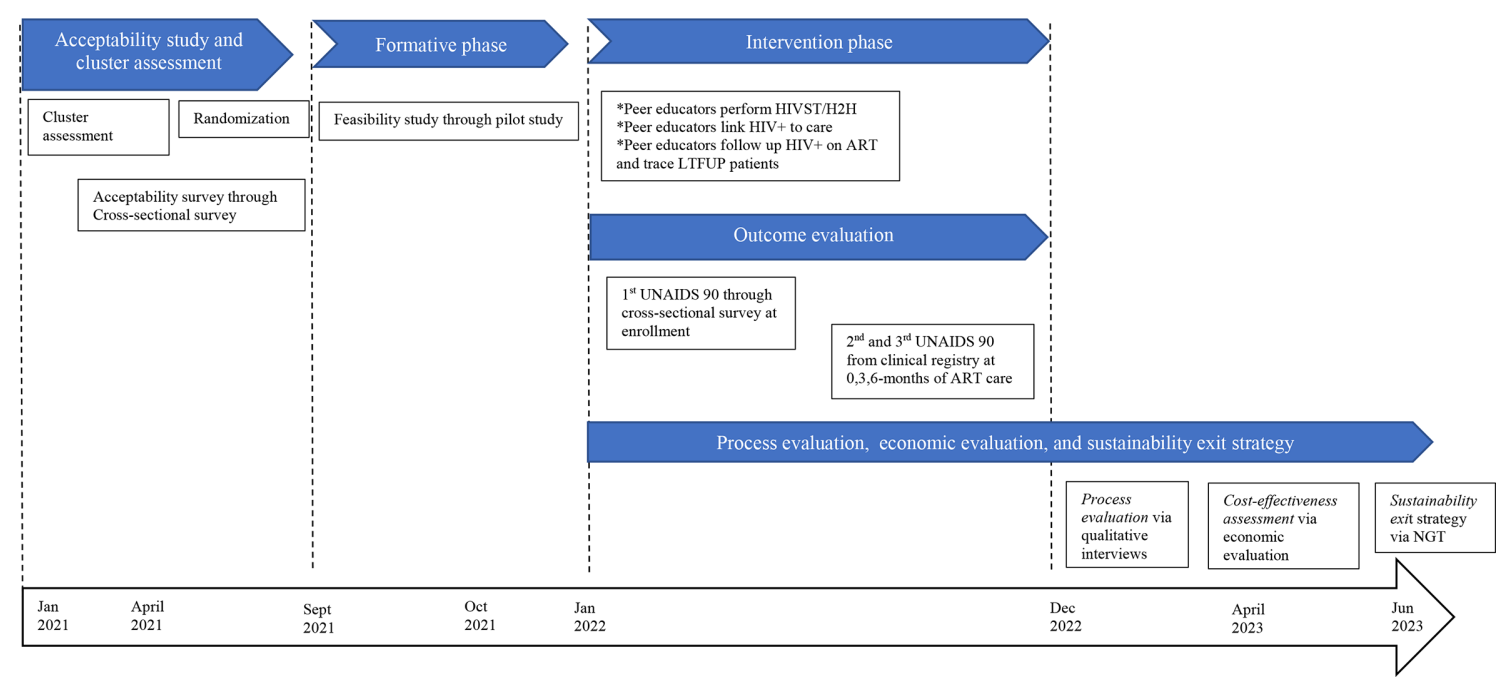

Figure 4 Trial timeline of the teach-test-link-trace (TTLT) study, Tigrai, Ethiopia. This figure presents the timeline of the overall trial. ART, antiretroviral therapy; $\mathrm{H} 2 \mathrm{H}$, peer educators-led pinprick house-to-house HIV testing; HIVST, peer educators-led OraQuick HIV self-testing; LTFU, lost-to-follow-up; UNAIDS, The Joint United Nations Programme on HIV/AIDS. 
comprising five-point Likert scale. Stigma will be measured using 12 items (and five-point Likert scale) HIVrelated stigma scale ${ }^{21}$ translated to Tigrigna. We will also explore stigma qualitatively through exploring barriers to accessing care, interactions with health workers and peer educators. Moreover, demography data, type of ART, WHO clinical staging, CD4 counts, viral load, functional status and tuberculosis ( $\mathrm{Tb}) / \mathrm{HIV}$ coinfection status will also be extracted.

\section{Process evaluation}

In this phase, we will collect data for safety endpoints and other issues related to the quality of the interventions. In-depth interviews will be conducted by experienced and trained research assistant at the end of the baseline survey and 6 months visit time. Baseline in-depth interviews aim at exploring experiences with HIVST, $\mathrm{H} 2 \mathrm{H}$ and peer educators. At 6-month follow-up, experiences with peer educators in retention in care and challenges to HIV care continuum will be explored. In particular, we will ask additional questions to participants in the HIVST study arms about their experiences with the HIVST kit, associated adverse effects such as violence, unintentional disclosure and self-harm. We will also ask all participants about perceived likelihood of using HIVST in the future and if they would be happy to be tested by peer educators during home-based HIV testing. Furthermore, we will ask participants in the intervention arm about their experiences with the peer educators. All in-depth interviews in the process evaluation will be audio-recorded.

\section{Economic evaluation}

In this phase, we will record all resources which will be used by those randomised to each study arm, and then cost each resource use item to estimate the total costs. We will collect financial and economic data to undertake a cost consequence in which the costs associated with HIV counselling, testing, linking and tracing will be estimated for control and experimental groups. Costs of HIV treatment will also be estimated for both groups. Differences in costs between the groups will be presented alongside differences in the primary and secondary outcomes.

\section{Sustainability exit strategy}

In this phase, we will organise a workshop to develop the strategies for the sustainability of the intervention. A consensus-based study called NGT will be applied to discuss the findings, consult on how to sustain and scale up the programme, and develop a sustainability and scalability of the project. The following experts will be included in the workshop: researchers, programme managers in governmental and non-governmental organisations, and health workers. The sustainability of the programme will be assessed using a HIV/AIDS Programme Sustainability Analysis Tool through gap analysis. ${ }^{25}$ The analysis tool briefly includes understanding the scalability and quantifying the unit cost of the programme, define targeted policy scenarios, quantify financial and human resources for the policy scenarios, perform gap analysis and suggest modality to fill the gap.

\section{Statistical analysis}

Quantitative data analysis

We will describe the sociodemographic characteristics of the study participants by study arms and compare them using $\mathrm{t}$-test and $\chi^{2}$ tests to check for any imbalance between arms. Treatment effects will be assess using intention-totreat analysis with multilevel models. Multiple imputation will be performed if there is more than $10 \%$ data missing. The analysis approach will account for the clustering of individuals within kebeles with both an unadjusted and adjusted analysis to account for residual confounding. We will also compare complete case analysis results with imputed missing data as a sensitivity analysis. Analysis will be performed before unblinding the data.

\section{Qualitative data analysis}

We will analyse the qualitative data using thematic framework analysis. Initially, we will familiarise with each interview and then transcribe the audiotaped interviews so as to identify significant statements from the transcripts. We will then develop a codebook to develop codes, subsequent subthemes and themes inductively and deductively. We will use NVivo to perform open coding for the illustrative quotes. Finally, we will develop a working analytical framework, perform charting data into the framework matrix and subsequently interpret the data.

\section{Cost-effectiveness analysis and cost-utility analysis}

In addition to the quantitative analysis described above, we will present the cost differences between the trial groups alongside differences in the primary and secondary endpoints. Sensitivity analyses will be undertaken to describe the uncertainty around the mean study results. We will also analyse the cost-utility analysis described through the number of life years saved adjusted to the account of loss of quality or for disability (qualityadjusted life years or disability-adjusted life years) for the secondary outcome.

\section{NGT analysis}

The sustainability exit strategy will be analysed using the HAPSAT V.2.0 software. The software enables us to (1) describe and quantify target population for activities; (2) specify activities; (3) quantify cost of clinical activities such as ART regimens, other HIV treatment-related medications, laboratory services, peer educators and health workers labour, and other non-clinical activities such as waiting time and transportation cost; (4) specify policy scenarios; (5) visualise of the analysis of the policy scenarios in figures and summary tables; and finally (6) choose the policy. We will apply a content analysis to synthesise the qualitative information of the NGT.

\section{Application of the Theory of Change in TTLT model}

The TTLT trial will be guided by the Theory of Change (ToC). ${ }^{26}$ ToC, originally incepted by Connel Weiss, ${ }^{27}$ 
Table 2 Application of Theory of Change in TTLT trial in Tigrai, Ethiopia, 2020

\begin{tabular}{|c|c|}
\hline Component of ToC & Description \\
\hline Impact & Reducing AIDS epidemic in Tigrai \\
\hline Outcomes & $\begin{array}{l}\text { Primary outcomes: UNAIDS first } 90 \text {, second } 90 \text { and third 90; } \\
\text { Secondary outcomes: QoL and stigma }\end{array}$ \\
\hline Interventions & $\begin{array}{l}\text { HIVST, } \mathrm{H} 2 \mathrm{H}, \mathrm{HIVST} \text { or } \mathrm{H} 2 \mathrm{H} \text {; and } \\
\text { referral of HIV diagnosed people to treatment linkage, follow-up of patients on ART, and trace of LTFU } \\
\text { patients }\end{array}$ \\
\hline Assumptions & $\begin{array}{l}\text { Peer educators situated in health facilities } \\
\text { Peer educators able to provide home-based pinprick HIV testing and self-HIV testing services and } \\
\text { refer to health facilities } \\
\text { Individuals are willing to accept self-led and peer-led finger prick HIV testing }\end{array}$ \\
\hline
\end{tabular}

$\mathrm{H} 2 \mathrm{H}$, peer educators-led pinprick house-to-house HIV testing; HIVST, peer educators-led OraQuick HIV self-testing; LTFU, lost-to-follow-up; QoL, quality of life; UNAIDS, The Joint United Nations Programme on HIV/AIDS.

articulates the change in impact of an intervention through intermediate and long-term outcomes through a complex causal pathway under certain conditions or assumptions. ${ }^{28}$ ToC has the following components: impact, long-term outcomes, intermediate outcomes, interventions, rationale, assumptions and indicators. Table 2 outlines these components in the context of the current study's model. Guided by the theory of change, we theorise that peer-led $\mathrm{H} 2 \mathrm{H}$ or HIVST would lead to improved knowledge of HIV status by lessening the barriers to HIV testing. For example, the HIVST may reduce perceived or enacted HIV-related stigma from the community or from the health workers as it allows people to test themselves in a private place, leading to improved uptake of HIV testing, reduce time to linkage to HIV care and improved clinical and immunological outcomes. Similar analogy also works for the impact of $\mathrm{H} 2 \mathrm{H}$ in knowledge of HIV status, linkage to care, and clinical and immunological outcomes. Nevertheless, there is also a possibility that the HIVST or H2H could not be successful, if people are still concerned that others (or family members in case of $\mathrm{H} 2 \mathrm{H}$ ) may discover about their HIV status.

\section{Dissemination plan}

We will disseminate findings of the study to (HIV) community, health workers and policy and programme managers. We plan a community meeting in selected kebeles in each zone to share the result. We will also organise different workshops with HIV experts and relevant stakeholders. We will prepare a policy brief to Tigrai Health Bureau, and full report to Flinders University and
Mekelle University. Finally, we will share our results with the scientific community through conference presentations, peer-reviewed publications and media releases.

\section{Data management}

Quantitative data will be collected using an interviewer administered questionnaire. The questionnaires and other related information to the study will be kept and locked in a filing cabinet with the key only accessible to the chief investigator. We will audio-record the qualitative interviews and will be transcribed and translated word-by-word. Once the transcription and translation are completed, we will destroy the audio recordings.

\section{Patient and public involvement}

Adult people with or without HIV will be involved in the trial.

\section{Data monitoring committee}

This trial will be led by the Tigrai-Flinders HIV research group comprising researcher from Flinders University in Australia and Mekelle University in Tigrai, Northern Ethiopia. The Tigrai-Flinders HIV Research Programme is a 5-year Australia's National Health and Medical Research Council (NHMRC)-funded project and aims to improve the entire HIV care continuum programme through peer educators and community health workers in Tigrai. Additionally, we will establish a monitoring and evaluation committee comprising an independent DMC to oversee the data collection processes in the study settings (local DMC), and an evaluation committee that will independently 
evaluate the overall progress of the project (international DMC). The local DMC members will comprise experts from Tigrai in HIV from zonal health department and district health office, HIV patients from people living with HIV (PLWHA) associations, researchers from Tigrai Research Institute, HIV programme managers from Tigrai Regional Health Bureau, leaders from community and board members from institutional review board of Mekelle University. The international DMC will be made up of experts from Ethiopian Ministry of Health, Ethiopian Public Health Institute, international nongovernmental organisations working in Tigrai, WHO and UNAIDS. This committee will monitor the overall progress of the trial-the implementation of the trial against the protocol, the importance to continue or stop the trial, overall quality of the intervention and success of the sustainability exit strategy.

For trial monitoring, the research team will report field work progress for review to the local DMC. Any issues or concerns related to adverse events as a result of the interventions will be reported as they happen and the local DMC will review these reports and take necessary measures, including enabling participants who may report adverse event have 24 hours access to counselling services. The Tigrai-Flinders Research group and the local DCM will meet monthly, and once every 6 months with the international DMC. We acknowledge that some trials with long duration of interventions may need interim analysis to assess progress and determine whether they should continue or not. However, as this is a short duration trial, formal statistical stopping rules have not been planned.

\section{DISCUSSION}

The success of HIV testing, UNAIDS's first 90, is the key for the success of the treatment linkage, retention in care and virological suppression, UNAIDS's second and third 90. The introduction of HIVST and H2H (through peer educators) is a novel intervention programme to achieve the HIV diagnosis target for the following reasons: (1) selftesting and home-based HIV testing reduce HIV related stigma, a key barrier to the entire HIV care continuum, ${ }^{729}$ as both services allow for the highest degree of privacy; (2) both services avoid structural barriers to HIV testing services such as distance, transportation fee and waiting times $^{7}$; and (3) the involvement of peer educators increase trust to HIV care system because they can share their lived experiences of HIV testing, treatment linkage and its benefit, and retention in care.

The TTLT model is an alternative intervention that combines community-based and facility-based services. The overall goal of the intervention is to contribute to ending AIDS epidemic by 2030 through improving the HIV testing and diagnosis, treatment linkage, and clinical, immunological and virological outcomes. Working with the Tigrai Regional Health Bureau, Tigrai, Ethiopia and NHMRC, Australia, the trial is designed to answer the following questions: (1) Is home-based peer-led HIV self-testing and immediate HIV testing acceptable? (2) Is home-based peer-led HIV self-testing and immediate HIV testing effective? And (3) Is home-based peer-led HIV selftesting and immediate HIV testing improve ART linkage and virological suppression?

The first research question will be addressed through a cross-sectional survey to measure acceptability of intervention. By assessing the acceptability of the model, we will be able to know how many people who accepted the intervention will be tested, and willing to remain in the peer-led HIV care and treatment. Furthermore, as we will ask 3 months' HIV testing history, we will be able to measure the baseline data of the proportion of people who know their HIV status. This finding will be compared with after intervention findings which will be known by answering the second research question. The second research question will be addressed through providing the HIVST and/or H2H interventions. In these interventions, we will assess whether these interventions improve testing uptake or not. Coupled with qualitative exploration of experiences on service uptake with individuals in the trial and the cost-effectiveness analysis, we will measure a robust evidence on the effectiveness of the model.

Although acceptability (question 1) and testing uptake (question 2) are an important piece of evidence for HIV testing policy, the design of this study allows for participants to be linked to nearby (or their preferred) health facilities for confirmatory test. Additionally, participants will be linked to ART care with the help of peer educators and will follow them to remain in the care. This will help us to answer question 3. In answering questions 2 and 3, we will assess the impact of the peer-led services to the entire HIV care continuum and this subsequently contributes to the ultimate goal of the UNAIDS goal, virological suppression and ending the AIDS epidemic.

The fact that this study begins with community-based as opposed to facility-based is a major strength of the trial. The cluster randomised trial nature of the study allows to extrapolate the population benefit of the trial. The community-based HIVST and $\mathrm{H} 2 \mathrm{H}$ interventions will allow individuals who never sought care to link to care, a novel gateway to improve the knowledge of HIV status. ${ }^{9}$ Such early diagnosis will improve the benefit and effectiveness of ART. The HIVST and H2H programmes can also be integrated with contact tracing, one of the public health measures related to COVID-19, which will be another opportunity to support the already compromised facility-based HIV testing services as a result of the COVID-19-related restrictions.

The study may have the following limitations. First, there may be a partner violence should their partner found out the self-testing in general and HIV positive result in particular. To address this, we will: (1) explain to the study participants before the start of the intervention, (2) inform them to report to us (or other responsible body) should any associated violence happen, and 
(3) inform them the availability of a 24-hour counselling through telephone or in person. Second, there may be safety concerns of stress, anxiety or self-harm associated with knowing the positive HIV status, a possible consequence which could also be happening in the standard HIV testing services. As we have described elsewhere in the document, the UNAIDS found no evidence of these issues associated with self-testing or home-based HIV testing. ${ }^{30}$ In addition to counselling, participants will be informed to contact peer educators or call to nearby health facilities any time. Third, the clusters are small and the randomisation process will not be adequate to reduce the sampling bias. To minimise this, we stratified the clusters by zones at the design stage.

In conclusion, it is expected that the project will have a substantial contribution to early HIV care presentation, retention in care and the virological suppression. We expect that findings of this project will produce knowledge, build capacity and inform policy and practice.

\section{ETHICS AND DISSEMINATION}

Ethics approval (EC00188) was obtained from the Southern Adelaide Clinical Health Research Ethics Committee of South Australia. We will receive permissions from respective institutions and written consents from the participants prior to interview and testing. Participants will be informed to withdraw at any stage of the trial should they will not be willing to continue. The research project has a direct benefit for the study participants as every participant will know their HIV status and will be linked to care, if diagnosed with HIV. Although none was reported by UNAIDS, there may be minimal risks or adverse events during the HIVST/H2H process, such as emotional disturbance, self-harm, violence or feel burden due to their involvement. If participants feel any negative concern related to the study, the following measures will be taken: (1) participants will be informed to discontinue the study at any level should they feel uncomfortable; and (2) participants will be provided a contact address of the peer educator and referred to a counselling service within the respective health facilities. For the NGT participants, we will seek written consent from participants prior to recording the discussion. We will also assure all panellists that their information will be treated with the strictest confidence and no identifying background information will be published. In order to disseminate findings of the project, we will publish them in peer-reviewed journals, present on workshops and conferences, and share through a media release.

\section{Twitter Hailay Gesesew @Gesesew and Jonathan Karnon @jonkarnon}

Acknowledgements We acknowledge the Australian National Health and Medical Research Council (NHMRC) for funding the project. The funding organisation has no role in study design; collection, management, analysis and interpretation of data; writing of the manuscript; and the decision to submit the manuscript for publication.

Contributors HG, PW, JK, RW and LM have been involved in conceiving and designing the protocol. HG was involved in developing the initial draft and PW, JK,
RW and LM critically reviewed the manuscript. HG, PW, JK, RW and LM read and approve the final manuscript. $\mathrm{HG}$ received the fund.

Funding Australian National Health and Medical Research council (NHMRC) (Grant code: GNT1176076).

Map disclaimer The depiction of boundaries on this map does not imply the expression of any opinion whatsoever on the part of BMJ (or any member of its group) concerning the legal status of any country, territory, jurisdiction or area or of its authorities. This map is provided without any warranty of any kind, either express or implied.

\section{Competing interests None declared.}

Patient and public involvement Patients and/or the public were not involved in the design, or conduct, or reporting, or dissemination plans of this research.

Patient consent for publication Not required.

Provenance and peer review Not commissioned; externally peer reviewed.

Open access This is an open access article distributed in accordance with the Creative Commons Attribution Non Commercial (CC BY-NC 4.0) license, which permits others to distribute, remix, adapt, build upon this work non-commercially, and license their derivative works on different terms, provided the original work is properly cited, appropriate credit is given, any changes made indicated, and the use is non-commercial. See: http://creativecommons.org/licenses/by-nc/4.0/.

ORCID iD

Hailay Gesesew http://orcid.org/0000-0002-3531-4400

\section{REFERENCES}

1 TahF, LaithA-R, DireslgnA, et al. Global, regional, and national incidence, prevalence, and mortality of HIV, 1980-2017, and forecasts to 2030, for 195 countries and territories: a systematic analysis for the global burden of diseases, injuries, and risk factors study 2017. Lancet HIV 2019;6:e831-59.

2 CSA, ICF. Ethiopia demographic and health survey 2016: HIV report. Addis Ababa, Ethiopia, and Rockville, Maryland, USA Central Statistical Agency (CSA) and ICF; 2018.

3 Deribew A, Biadgilign S, Deribe K, et al. The burden of HIV/AIDS in Ethiopia from 1990 to 2016: evidence from the global burden of diseases 2016 study. Ethiop J Health Sci 2019;29:859-68.

4 UNAIDS. UNAIDS 90-90-90: an ambitious treatment target to help end the AIDS epidemic. Geneva, Switzerland; 2014.

5 Gesesew HA, Ward P, Woldemichael K, et al. Hiv care continuum outcomes: can Ethiopia meet the UNAIDS 90-90-90 targets? Ethiop $J$ Health Sci 2020;30:179-88.

6 UNAIDS. Understanding fast-track: accelerating action to end the AIDS epidemic by 2030. Geneva, Switzerland UNAIDS; 2015: 2.

7 Gesesew H, Ward P, Woldemichael K, et al. Improving the UNAIDS 90-90-90 treatment targets: solutions suggested from a qualitative study of HIV patients, community advocates, health workers and program managers in Jimma, Southwest Ethiopia. Int J Environ Res Public Health 2020;17:378.

8 MSF/UNAIDS. Closer to home: delivering antiretroviral therapy in the community: experience from four countries in southern Africa; 2010: 18.

9 Oldenburg CE, Ortblad KF, Chanda MM, et al. Zambian peer educators for HIV Self-Testing (ZEST) study: rationale and design of a cluster randomised trial of HIV self-testing among female sex workers in Zambia. BMJ Open 2017;7:e014780.

10 Chanda MM, Ortblad KF, Mwale M, et al. HIV self-testing among female sex workers in Zambia: a cluster randomized controlled trial. PLoS Med 2017; 14:e1002442.

11 Ortblad KF, Chanda MM, Musoke DK, et al. Acceptability of HIV self-testing to support pre-exposure prophylaxis among female sex workers in Uganda and Zambia: results from two randomized controlled trials. BMC Infect Dis 2018;18:503.

12 Moshoeu MP, Kuupiel D, Gwala N, et al. The use of home-based HIV testing and counseling in low-and-middle income countries: a scoping review. BMC Public Health 2019;19:132.

13 WHO. Doing it for themselves: Peer-led HIV testing in Viet Nam improves access to care, 2017. Available: https://www.who.int/ westernpacific/news/feature-stories/detail/doing-it-for-themselvespeer-led-hiv-testing-in-viet-nam-improves-access-to-care [Accessed 12 Mar 2020].

14 Yan H, Zhang R, Wei C, et al. A peer-led, community-based rapid HIV testing intervention among untested men who have sex with men in 
China: an operational model for expansion of HIV testing and linkage to care. Sex Transm Infect 2014;90:388-93.

15 FDRE. Health sector development program IV 2010/11 - 2014/15. Addis Ababa Minstory of Health; 2010.

16 TRHB. Tigray regional health bureau II ten years health Bulletin (EFY 1998-2007 or 2006/7-2014/5). Mekele, Tigray, Ethiopia Tigray Regional Health Bureau (TRHB); 2016.

17 Sekhon M, Cartwright M, Francis JJ. Acceptability of healthcare interventions: an overview of reviews and development of a theoretical framework. BMC Health Serv Res 2017;17:88.

18 WHO. WHO prequalification of in vitro diagnostics (public Reort); product: OraQuick HIV self-test; 2019.

19 Meshesha HM, Nigussie ZM, Asrat A, et al. Determinants of virological failure among adults on first-line highly active antiretroviral therapy at public health facilities in Kombolcha town, northeast, Ethiopia: a case-control study. BMJ Open 2020;10:e036223.

20 Tesfay A, Gebremariam A, Gerbaba M, et al. Gender differences in health related quality of life among people living with HIV on highly active antiretroviral therapy in Mekelle town, Northern Ethiopia. Biomed Res Int 2015;2015:1-9.

21 Reinius $M$, Wettergren L, Wiklander M, et al. Development of a 12-Item short version of the HIV stigma scale. Health Qual Life Outcomes 2017;15:115.

22 Hayes RJ, Bennett S. Simple sample size calculation for clusterrandomized trials. Int J Epidemiol 1999;28:319-26.

23 Haileamlak A, Abraham $\mathrm{H}$. Will Ethiopia achieve the global target of 90-90-90? Ethiop J Health Sci 2019;29:298.
24 Whitehead AL, Julious SA, Cooper CL, et al. Estimating the sample size for a pilot randomised trial to minimise the overall trial sample size for the external pilot and main trial for a continuous outcome variable. Stat Methods Med Res 2016;25:1057-73.

25 UNAIDS. HIV/AIDS Program Sustainability Analysis Tool (HAPST) 2.0 Washington: USAIDS; 2011 [cited 202023 March]. Available from. Available: https://www.hfgproject.org/hivaids-program-sustainabilityanalysis-tool-hapsat-2-0/ [Accessed 23 March 2020].

26 Funnell S, Rogers P. Purposeful program theory: effective use of theories of change and logic models. Weily: Research Methods for the Social Sciences, 2011.

27 Connell W. Nothing as practical as good theory: exploring theorybased evaluation for comprehensive community initiatives for children and families. In: Connell W, ed. New approaches to evaluating community initiatives: concepts, methods, and contexts. Washington, DC: Aspen Institute, 1995: 65-92.

28 Paina L, Wilkinson A, Tetui M, et al. Using theories of change to inform implementation of health systems research and innovation: experiences of future health systems Consortium partners in Bangladesh, India and Uganda. Health Res Policy Syst 2017; 15:109.

29 Gesesew H, Lyon P, Ward P, et al. "Our Tradition Our Enemy": A Qualitative Study of Barriers to Women's HIV Care in Jimma, Southwest Ethiopia. Int J Environ Res Public Health 2020;17:833.

30 UNAIDS. A short technical update on self-testing for HIV. Geneva, Switzerland; 2014. 